\title{
CALiPER Exploratory Study: Accounting for Uncertainty in Lumen Measurements
}

Rolf Bergman, Rolf Bergman Consulting Mia Paget, PNNL (contract administrator) Eric Richman, PNNL (contract administrator)

March 2011

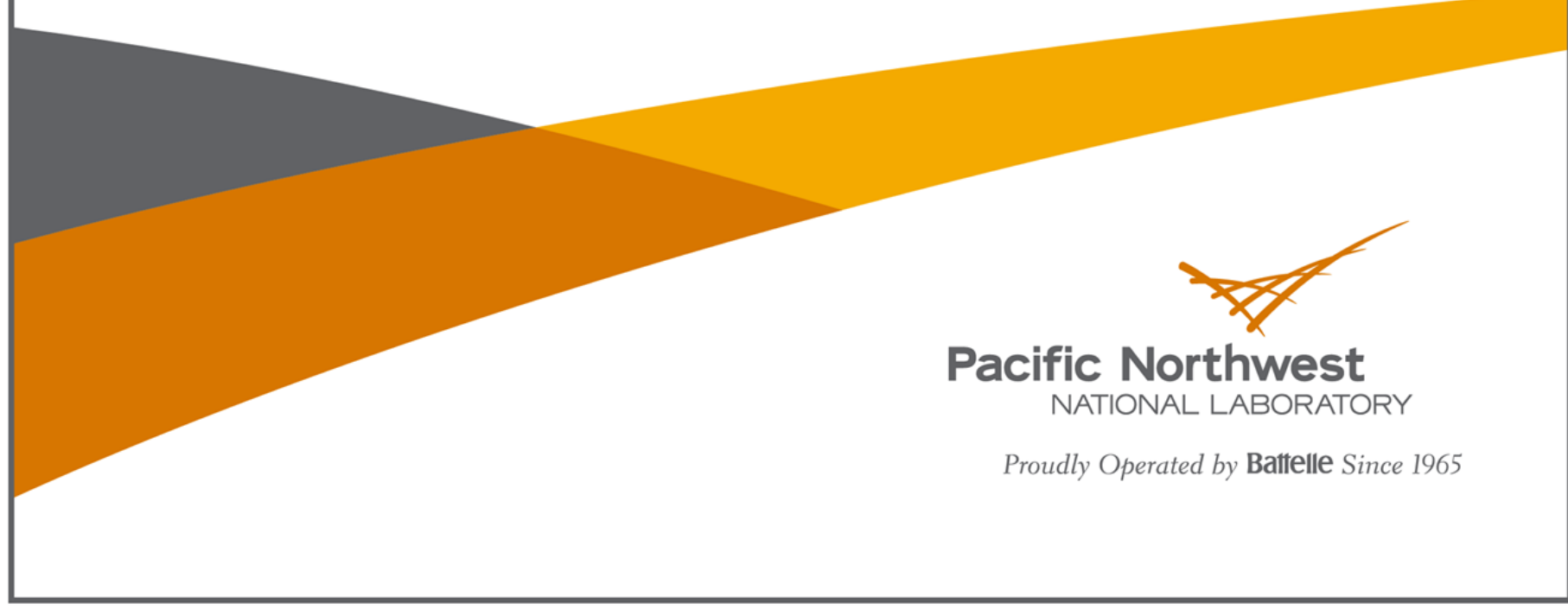




\title{
DISCLAIMER
}

This report was prepared as an account of work sponsored by an agency of the United States Government. Neither the United States Government nor any agency thereof, nor Battelle Memorial Institute, nor any of their employees, makes any warranty, express or implied, or assumes any legal liability or responsibility for the accuracy, completeness, or usefulness of any information, apparatus, product, or process disclosed, or represents that its use would not infringe privately owned rights. Reference herein to any specific commercial product, process, or service by trade name, trademark, manufacturer, or otherwise does not necessarily constitute or imply its endorsement, recommendation, or favoring by the United States Government or any agency thereof, or Battelle Memorial Institute. The views and opinions of authors expressed herein do not necessarily state or reflect those of the United States Government or any agency thereof.

\author{
PACIFIC NORTHWEST NATIONAL LABORATORY \\ operated by \\ BATTELLE \\ for the \\ UNITED STATES DEPARTMENT OF ENERGY \\ under Contract DE-AC05-76RL01830
}

Printed in the United States of America

Available to DOE and DOE contractors from the

Office of Scientific and Technical Information,

P.O. Box 62, Oak Ridge, TN 37831-0062;

ph: (865) 576-8401

fax: $(865) 576-5728$

email: reports@adonis.osti.gov

\author{
Available to the public from the National Technical Information Service \\ 5301 Shawnee Rd., Alexandria, VA 22312 \\ ph: (800) 553-NTIS (6847) \\ email: orders@ntis.gov <http://www.ntis.gov/about/form.aspx> \\ Online ordering: http://www.ntis.gov
}

This document was printed on recycled paper. 
PNNL-20320

\section{CALiPER Exploratory Study: Accounting for Uncertainty in Lumen Measurements}

Rolf Bergman, Rolf Bergman Consulting

Mia Paget, PNNL (contract administrator)

Eric Richman, PNNL (contract administrator)

March 2011

Prepared for

the U.S. Department of Energy

under Contract DE-AC05-76RL01830

Work done by Rolf Bergman Consulting through contract with the U.S. Department of Energy's Pacific Northwest National Laboratory Contract \#11259

Pacific Northwest National Laboratory

Richland, Washington 99352 



\section{Summary}

With a well-defined and shared understanding of uncertainty in lumen measurements, testing laboratories can better evaluate their processes, contributing to greater consistency and credibility of lighting testing — a key component of the U.S. Department of Energy (DOE) Commercially Available LED Product Evaluation and Reporting (CALiPER) program. Reliable lighting testing is a crucial underlying factor contributing toward the success of many energy-efficient lighting efforts, such as the DOE GATEWAY demonstrations, Lighting Facts Label, ENERGY STAR ${ }^{\circledR}$ energy efficient lighting programs, and many others.

Uncertainty in measurements is inherent to all testing methodologies, including photometric and other lighting-related testing. Uncertainty exists for all equipment, processes, and systems of measurement in individual as well as combined ways. A major issue with testing and the resulting accuracy of the tests is the uncertainty of the complete process. Individual equipment uncertainties are typically identified, but their relative value in practice and their combined value with other equipment and processes in the same test are elusive concepts, particularly for complex types of testing such as photometry. The total combined uncertainty of a measurement result is important for repeatable and comparative measurements for light emitting diode (LED) products in comparison with other LED products and other technologies. This study provides a detailed and step-by-step method for determining uncertainty in lumen measurements, working closely with related standards efforts and key industry experts.

This report uses the structure proposed in the Guide to Uncertainty Measurements (GUM) for evaluating and expressing uncertainty in measurements. The steps of the procedure are described and a spreadsheet format adapted for integrating sphere and goniophotometric uncertainty measurements is provided for entering parameters, ordering the information, calculating intermediate values and, finally, obtaining expanded uncertainties. Using this basis and examining each step of the photometric measurement and calibration methods, mathematical uncertainty models are developed. Determination of estimated values of input variables is discussed. Guidance is provided for the evaluation of the standard uncertainties of each input estimate, covariances associated with input estimates and the calculation of the result measurements. With this basis, the combined uncertainty of the measurement results and finally, the expanded uncertainty can be determined. 



\section{Acknowledgments}

This exploratory study conducted within the context of the U.S. Department of Energy Commercially Available LED Product Evaluation and Reporting (CALiPER) program was made possible and funded by the American Recovery and Reinvestment Act of 2009.

Advanced studies of lighting testing would not be possible without the on-going work and engaged participation of CALiPER testing laboratories, standards development organizations (such as Bureau International des Poids et Mesures, the International Commission on Illumination and the Illuminating Engineering Society), and numerous industry experts. Specific thanks go to Cameron Miller and his colleagues at the National Institute of Standards and Technologies, and to a number of independent lighting testing laboratories: Intertek (Cortland, New York), Independent Test Laboratories (Boulder, Colorado), Lighting Sciences, Inc. (Scottsdale, Arizona), On-Spex Laboratories/CSA International (Alpharetta, Georgia), and Aurora International Test Laboratory (Aurora, Ohio). 



\section{Acronyms and Abbreviations}

$\begin{array}{ll}\text { BIPM } & \text { Bureau International des Poids et Mesures } \\ \text { CALiPER } & \text { Commercially Available LED Product Evaluation and Reporting } \\ \text { CCT } & \text { Correlated Color Temperature } \\ \text { CFL } & \text { compact fluorescent lamp } \\ \text { CIE } & \text { International Commission on Illumination (Commission International de } \\ & \text { l'Eclairage) } \\ \text { DOE } & \text { U.S. Department of Energy } \\ \text { GUM } & \text { Guide to Uncertainty Measurements } \\ \text { IES } & \text { Illuminating Engineering Society } \\ \text { LED } & \text { light emitting diode } \\ \text { NIST } & \text { National Institute of Standards and Technology } \\ \text { PNNL } & \text { Pacific Northwest National Laboratory } \\ \text { SSL } & \text { solid-state lighting }\end{array}$





\section{Contents}

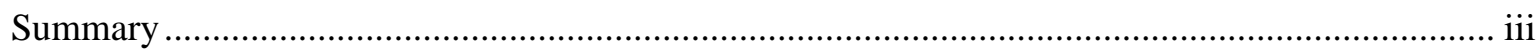

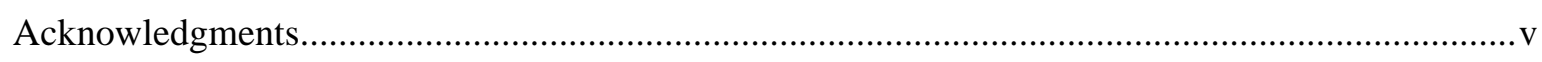

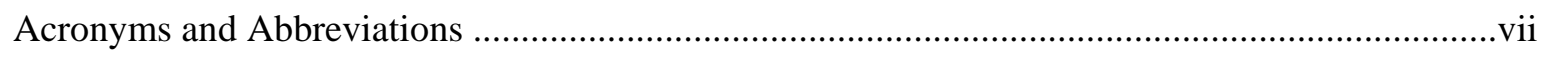

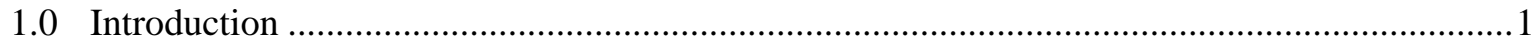

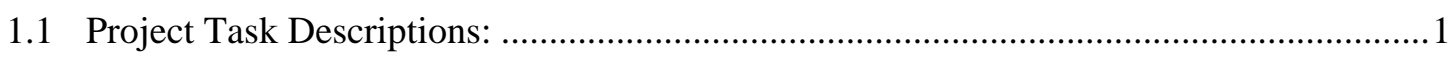

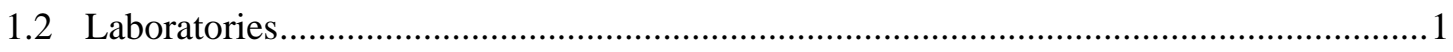

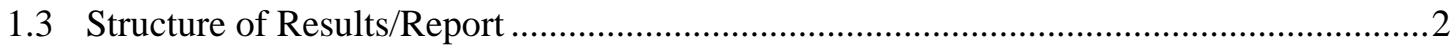

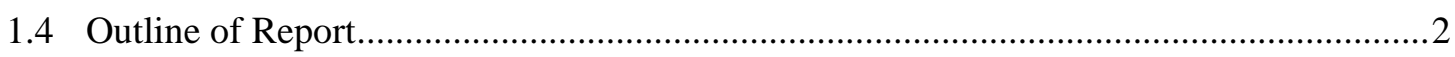

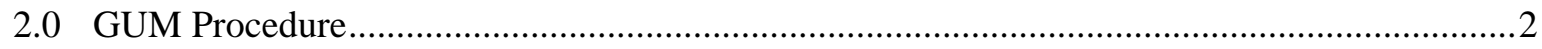

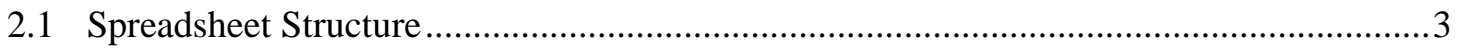

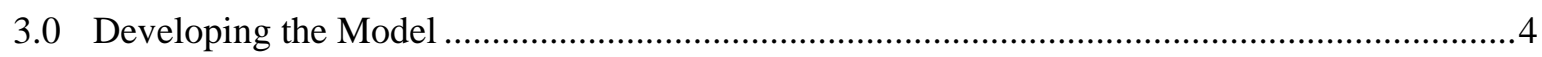

4.0 Determining the Estimated Value of the Input Quantity ....................................................6

4.1 Input Variables for an Integrating Sphere ....................................................................

4.1.1 Variable Estimates for the Reference Standard.....................................................

4.1.2 Variable Estimates for the Test Lamp ..............................................................

4.2 Input Variables for a Mirror Goniometer .....................................................................

4.2.1 Variable Estimates for the Reference Intensity Standard......................................9

4.2.2 Variable Estimates for the Test Lamp on a Goniometer ......................................10

5.0 Evaluate the Standard Uncertainties of Each Input Estimate ................................................11

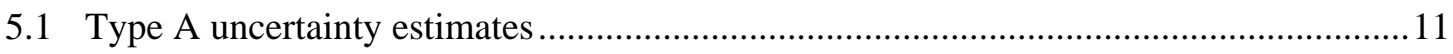

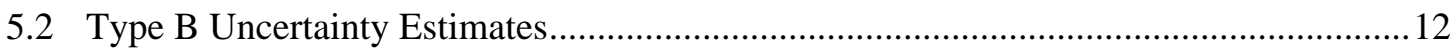

6.0 Evaluate the Covariances Associated With Input Estimates ................................................12

7.0 Calculate the Result of Measurement ..............................................................................12

8.0 Determine the Combined Uncertainty of the Measurement Results .....................................13

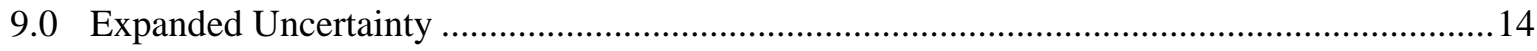

10.0 Reporting the Results Together With Combined Uncertainty ….........................................15

Appendix Accompanying Excel Spreadsheet ........................................................................ 1 



\subsection{Introduction}

Uncertainty in measurements is a necessary condition of all testing methodologies, including photometric and other lighting-related testing. Uncertainty exists for all equipment, processes, and systems of measurement in individual as well as combined ways. A major issue with testing and the resulting accuracy of the tests is the uncertainty of the complete process. Individual equipment uncertainties are typically identified, but their relative value in practice and their combined value with other equipment and processes in the same test are elusive concepts. The total combined uncertainty of a measurement result is important for repeatable and comparative measurements for light emitting diode (LED) products in comparison with other technologies as well as competing products.

This report describes the work completed to fulfill a subcontract project aimed at defining uncertainty related to lamp measurements for use by individual labs and others.

\subsection{Project Task Descriptions:}

- Develop a standard format for the documentation and reporting of uncertainty budgets for lumen measurements developed for each laboratory. Work with Pacific Northwest National Laboratory (PNNL) staff to ensure the format will work with expected needs of Energy Star, Commercially Available LED Product Evaluation and Reporting (CALiPER), and other U.S. Department of Energy (DOE) programs where measurement uncertainty is critical.

- Develop a set of uncertainty factors for the lamp standards developed and provided by National Institute of Standards and Technology (NIST) to testing laboratories. Factors shall be developed for LED standards as well as standards for lamps typically replaced by common LED products.

- Work with at least four testing laboratories to develop uncertainty budgets for solid-state lighting (SSL) products. Budgets will be developed for measurements taken for both integrating sphere systems and goniometer systems when they exist. Each uncertainty budget shall include a prioritized list of uncertainty factors and estimates of the size of uncertainty for each factor based on the laboratories’ specific equipment and measurement protocol characteristics.

- Prepare a written report based on visits to the laboratories that details the uncertainty budget values (Lamp Standards, plus laboratory equipment and systems) and their appropriate application to various testing scenarios. The reports shall be made available to the testing laboratory and the DOE.

\subsection{Laboratories}

A list of potential laboratories for site visits were identified by PNNL staff. Each was chosen based on its potential to represent the variety of laboratory types including sphere and goniometer testing capability and level of testing experience. Over a six month period, each of these five laboratories was visited to gain insight on laboratory operations to help in developing uncertainty budgets:

- Intertek, Cortland, New York

- Independent Test Laboratories, Boulder, Colorado

- Lighting Sciences, Inc., Scottsdale, Arizona

- On-Spex Laboratories (CSA International), Alpharetta, Georgia 
- Aurora International Test Laboratory, Aurora, Ohio

\subsection{Structure of Results/Report}

Prior to visiting any of the laboratories, a format for the uncertainty budget was worked with Cameron Miller from NIST. With his concurrence, the following process was adopted:

- Use the structure proposed in the Guide to Uncertainty Measurements (GUM) beginning with a model equation. (See reference below.)

- Use a spreadsheet format being developed by CIE TC 2-43, which is also used by NIST and other calibration labs in their calibration reports, for recording the values of the variables involved, the uncertainty of each variable, how that uncertainty is deduced (Type A or B), and the sensitivity coefficient for each variable uncertainty. ${ }^{1}$ From the spreadsheet the value of the lumen output is easily calculated along with an expanded uncertainty in the lumen value.

- Review the resulting spreadsheet format with each laboratory visited and work with the lab in an attempt to obtain values that the laboratories would use; at least show them how to obtain the variable estimates and the uncertainties needed.

\subsection{Outline of Report}

The lumen uncertainty report consists of two parts, this written report and the accompanying Excel spreadsheet which contains three lumen uncertainty worksheets, two for integrating sphere measurements, each with a different measurement system in mind, and one spreadsheet for goniometer measurements. In addition the eight-step GUM structure is noted on the first page of the workbook and a worksheet contains an abbreviated procedure of how the GUM steps are applied to obtain the active worksheets.

The rest of this report explains the structure of the worksheets and discusses issues involved.

\subsection{GUM Procedure}

As mentioned above this report uses the structure proposed in GUM for evaluating and expressing uncertainty in measurements. The 2008 version of the GUM (JCGM 100-2008) is available for free download from the Bureau International des Poids et Mesures (BIPM) website, www.bipm.org/en/publications/guides/gum.html. At the end of the main section of the GUM, section 8 summarizes the procedures for evaluating and expressing uncertainty in an eight-step procedure as follows:

1. Express mathematically the relationship between the measurand $Y$ and the input quantities $X_{\mathrm{i}}$ on which $Y$ depends: $Y=f\left(X_{1}, X_{2}, \ldots, X_{\mathrm{N}}\right)$. The function $f$ should contain every quantity, including all corrections and correction factors that can contribute a significant component of uncertainty to the result of the measurement (see 4.1.1 and 4.1.2).

\footnotetext{
${ }^{1}$ The International Commission on Illumination Technical Committee, CIE TC 2-43 encompasses Determination of Measurement Uncertainties in Photometry, http://div2.cie.co.at/?i_ca_id=561\&pubid=251.
} 
2. Determine $x_{\mathrm{i}}$, the estimated value of input quantity $X_{\mathrm{i}}$, either on the basis of the statistical analysis of series of observations or by other means (see 4.1.3).

3. Evaluate the standard uncertainty $u\left(x_{\mathrm{i}}\right)$ of each input estimate $x_{\mathrm{i}}$. For an input estimate obtained from the statistical analysis of series of observations, the standard uncertainty is evaluated as described in 4.2 (Type A evaluation of standard uncertainty). For an input estimate obtained by other means, the standard uncertainty $u\left(x_{\mathrm{i}}\right)$ is evaluated as described in 4.3 (Type B evaluation of standard uncertainty).

4. Evaluate the covariances associated with any input estimates that are correlated (see 5.2).

5. Calculate the result of the measurement, that is, the estimate $y$ of the measurand $Y$, from the functional relationship $f$ using for the input quantities $X_{\mathrm{i}}$ and the estimates $x_{\mathrm{i}}$ obtained in step 2 (see 4.1.4).

6. Determine the combined standard uncertainty $u_{\mathrm{c}}(y)$ of the measurement result $y$ from the standard uncertainties and covariances associated with the input estimates, as described in Clause 5 . If the measurement determines simultaneously more than one output quantity, calculate their covariances (see 7.2.5, H.2, H.3, and H.4).

7. The purpose of an expanded uncertainty $U$ is to provide an interval $y-U$ to $y+U$ that may be expected to encompass a large fraction of the distribution of values that could reasonably be attributed to the measurand $Y$. If it is necessary to give an expanded uncertainty $U$, multiply the combined standard uncertainty $u_{\mathrm{c}}(y)$ by a coverage factor $k$, typically in the range 2 to 3 , to obtain $U=k \bullet u_{\mathrm{c}}(y)$. Select $k$ on the basis of the level of confidence required of the interval (see 6.2, 6.3, and especially Annex $\mathrm{G}$, which discusses the selection of a value of $k$ that produces an interval having a level of confidence close to a specified value).

8. Report the result of the measurement y together with its combined standard uncertainty $u_{c}(y)$ or expanded uncertainty $U$ as discussed in 7.2.1 and 7.2.3; use one of the formats recommended in 7.2.2 and 7.2.4. Describe, as outlined in Clause 7, how $y$ and $u_{\mathrm{c}}(y)$ or $U$ were obtained.

In the sections below, the above procedure is applied to lumen measurements from integrating spheres and goniometers using Excel spreadsheets that detail the uncertainty of measurements for various measurement techniques.

\subsection{Spreadsheet Structure}

The input quantities/variables needed to obtain the lumen output for a given system and lamp type are placed in the spreadsheet one variable per row. In each worksheet there are two equations broken out from the main model to make the main model less cumbersome in writing. These describe correction factors for the test and standard reference lamp, respectively. The two correction factor model equations are placed at the top and accounted for separately. The variables of the third model equation are then listed starting with the summary values of the correction equations incorporated first.

In the columns the following information is recorded:

- name of variable

- the symbol used for the variable

- units 
- estimated value of the variable (as discussed in the last section)

- the estimated uncertainty

- uncertainty type

- degrees of freedom

- sensitivity coefficient

- contribution of the uncertainty to the lumen output, and

- relative contribution of the uncertainty.

This ordering of the information obtained allows calculation of the lumen output (luminous flux) of the test lamp at the bottom and the expanded uncertainty. As mentioned in the Introduction, this format has been adopted by CIE TC-43 and now NIST and other laboratories are using the format in reporting their standard lamp data on certificates.

\subsection{Developing the Model}

According to the GUM, a mathematical equation is written to describe the lumen measurement of a test lamp, taking into account the various factors that contribute uncertainty to the measurement. The luminous flux of a test lamp is the "measurand" or output variable. The symbol $\Phi$ is used for the luminous flux of the test lamp. Note that test lamp related input quantities will not have a subscript.

The flux is measured by a detector, either a "broadband" detector with a luminosity filter, whose output response (either voltage or current) is proportional to the lamp lumen flux, or a spectral detector where the output per pixel (a small wavelength range) is normally a quantity of charge. The amount of charge is proportional to the energy in a given wavelength region. To obtain lumen values the spectral power is multiplied by the luminosity function and summed. This is usually done automatically by manufacturers of spectral detector equipment using computer software. In either case, this electrical response for lumens is denoted by the letter " $y$."

The output response of the detector is normally calibrated using a lamp standard, denoted by subscript R, traceable to NIST or a National Metrology Institute if outside the United States. For integrating sphere measurements, reference standards can be obtained calibrated either as lumen standards, which specify the lumen output of the lamp when operating at a given current (usually direct current), or calibrated as total spectral flux, i.e., the radiant flux at given multiple wavelength intervals over the visible range. Again, the total spectral flux reference lamp is to be operated at a fixed current to provide this spectral output.

For goniometer measurements, lamp luminous intensity standards calibrated in candelas can be obtained from NIST. However, reference lamp standards calibrated for lumen output, often called face lumens, are also used for goniometers. Particularly, halogen-based reflector lamps, e.g., PAR 38 lamps are convenient. This or other types of lamps should have calibrated lumen values traceable to NIST or another calibration laboratory. There are advantages to using lumen reference standards over intensity standards; this will be discussed later. In all cases, the standard lamp comes with a certificate which gives the value of lumens (or spectral radiant flux) at a given current. The certificate also gives the input measurement factors and their associated uncertainty, from which the combined and expanded 
uncertainties of the standard reference lumen value are given. Normally, the expanded uncertainty is given at a coverage factor, $k$, of 2 .

As mentioned above, the standard reference lamp is specified to operate at a given current, $J_{R}$, and this current must be measured either using a calibrated ammeter or wattmeter with an internal resistor shunt or by the voltage across an external resistive shunt that has been calibrated. In the case of an external shunt, the voltage is measured using a calibrated voltmeter. Further, it is known from scientific principles that the lumen output of incandescent lamps changes with current at a rate of about seven times the change in current, while the lumen output changes with voltage at about four times the rate of voltage change.

Depending on the type of test lamp, the operating condition is usually a fixed voltage, usually AC. Again the test lamp voltage and/or current is measured using a calibrated meter. In cases where the test lamp is not an incandescent or halogen lamp, the dependence of the lumen output on the rate of change of input voltage depends very much on the type of lamp being tested. For SSL and compact fluorescent lamps (CFLs), it strongly depends on the type of ballast or driver used.

In summary, to this point in the discussion the input factors are as follows: lumen detector response, standard reference-lamp-rated lumens with specified electrical operating conditions and voltage/current measurements. These factors are incorporated into a mathematical model. Doing so, assuming a known resistor shunt for measuring the current of the reference lamp standard and a voltmeter to measure the test lamp operating voltage, we have the following:

$$
\Phi=\Phi_{\mathrm{R}} \cdot \frac{y}{y_{\mathrm{R}}} \cdot\left(\frac{U \cdot c}{U_{0}}\right)^{m_{\mathrm{U}}}\left(\frac{U_{\mathrm{R}} \cdot c}{J_{\mathrm{R}} \cdot R}\right)^{-m_{J_{\mathrm{R}}}}
$$

$\Phi_{\mathrm{R}}$ refers to the luminous flux of the reference standard when measured at a current of $J_{\mathrm{R}} \cdot y_{\mathrm{R}}$ refers to the electrical signal from the photometer that represents the raw electrical data of the luminous flux of the reference standard. In many systems where measurements are semi-automated $\Phi_{\mathrm{R}} / y_{\mathrm{R}}$ is the calibration factor for the integrating sphere or goniometer. When spectral total flux standards are used, then $\Phi_{\mathrm{R}} / y_{\mathrm{R}}$ can be understood as the ratio of the summed products of the spectral radiance and the luminosity function.

$\mathrm{U}$ is the reading of the input voltage of the test lamp, while $U_{0}$ is the reference voltage at which the lamp is to be tested, e.g., $120 \mathrm{~V}$. The " $c$ " in the above equation refers to the voltmeter calibration constant which is obtained from the meter calibration certificate as the fraction of the nominal value. The $m_{U}$ exponent expresses the relationship between a change in lumen output and the input voltage. If the lamp is an incandescent lamp then $m_{U}$ is on the order of 4 , while for well-ballasted CFL or SSL it may be very small, i.e., lumen output is not sensitive to changes in input voltage.

$U_{R}$ is the voltage across the resistor shunt, value R, (note that ' $R$ ' is also used as a subscript to refer to reference standard lamp values) due to the calibration current $J_{\mathrm{R}}$ passing through the resistor. The $m_{\mathrm{J}}$ exponent expresses the relationship between a change in lumen output and the reference lamp current. Since the reference lamp is usually an incandescent lamp then $m_{\mathrm{J}}$ is on the order of 7 .

Other additional defining factors that contribute to uncertainty need to be defined. Self-absorption corrections account for the spatial and spectral mismatch variations that need to be considered for 
integrating spheres. For spectral total flux standards the spectral mismatch is assumed to be negligible. Further there are temperature effects to be considered, particularly for fluorescent and SSL lamps. Thus the final model for spectral measurements of a test lamp in an integrating sphere, e.g., SSL lamp or fixture, consists of three equations as follows:

$$
\begin{gathered}
\operatorname{cor} S=\left(1+a \Delta T_{\alpha}-\Delta s f-\gamma \Delta t\right) \\
\operatorname{cor}_{\mathrm{R}}=\left(1+\alpha_{\mathrm{R}} \Delta T_{\alpha \mathrm{R}}-\Delta s f_{\mathrm{R}}-\gamma_{\mathrm{R}} \Delta t_{\mathrm{R}}\right) \\
\Phi=\Phi_{\mathrm{R}} \cdot \frac{y}{y_{\mathrm{R}}} \cdot \frac{y_{\mathrm{AR}}}{y_{\mathrm{A}}} \cdot\left(\frac{U \cdot c_{\mathrm{U}}}{U_{0}}\right)^{m_{\mathrm{U}}} \cdot\left(\frac{U_{\mathrm{R}} \cdot c_{\mathrm{R}}}{R \cdot J_{\mathrm{R}}}\right)^{m_{J_{\mathrm{R}}}} \cdot \frac{\operatorname{cor} S_{\mathrm{R}}}{\operatorname{cor} S}
\end{gathered}
$$

Notice that we have split out parts of the model equation, 2c, into two sub-equations, 2a and $2 \mathrm{~b}$ as discussed earlier.

For goniometers there are no self-absorption or spatial mismatch uncertainties, but spectral mismatch errors still exist. However, uncertainties in positioning of the source, both distance and angle, and introduction of stray light are new factors that add to the uncertainty.

Changes due to ageing of the lamp, particularly the reference standard, need to be considered for any type of measurement. Possibly other factors need to be added for a given measurement situation that is not discussed here.

\subsection{Determining the Estimated Value of the Input Quantity}

According to the GUM, the next step (Step 2) after building a mathematical model is to determine the estimated values of the input variables. For the detector input values (various " $y$ " factors), the estimated value is obtained as the average value of repeat measurements. In this way the factor variance or standard deviation is also obtained. This is called a Type A estimate since it is measured directly. How many repeat measurements need to be performed depends on how difficult it is to measure the variable, but it is suggested that a minimum of 10 measurements be made. (The reason that the minimum should be as many as possible is that the number of degrees of freedom for a variable is equal to the number of measurements minus one, and the relationship between the confidence level of a measurement and the coverage factor depends on the degrees of freedom.)

Some values have already been determined and do not need to be measured. The reference standard lumen value and the current at which it is to be operated are given in the certificate accompanying the standard. Also the volt/ wattmeter calibration " $c$ " factor is given in the certificate accompanying the instrument calibration; similarly for shunt resistors. In addition the combined uncertainties of these values are given in the certificate. This is called a Type B estimate since it was obtained from an outside source and not measured directly. For Type B inputs the number of degrees of freedom is considered infinite.

Other input variables cannot easily be measured so historical information, measurements by other labs of similar equipment, or an estimate, is used to provide the value of the variable. This is particularly true for variables that are small perturbations from zero, or from a normalized value of one. 
Using the above discussion as a guide the input variables that impact lumen measurement values either for an integrating sphere or a goniometer are listed below.

\subsection{Input Variables for an Integrating Sphere}

\subsubsection{Variable Estimates for the Reference Standard}

- Lumen Output, $\Phi_{\mathrm{R}}$ : given on the certificate.

- Current for operation, $J_{\mathrm{R}}$ : given on the certificate.

- Measurement of current, $U_{\mathrm{R}}$ : assumed to be done by measurement of voltage across a calibrated shunt resistor, $R$. The resistor value and uncertainty are listed on the resistor calibration certificate. The voltmeter has a center value c, close to the nominal reading, given on the calibration certificate. To obtain a Type A measurement, the $U_{\mathrm{R}}$ value should be measured repeatedly, a minimum of 10 times.

- Sensitivity of lumen flux to current, $m_{J R}$ : For incandescent or halogen lamps operating at a Correlated Color Temperature (CCT) near 2,856 K the change in lumen output with current can be expressed as an exponent of about 7. (Theoretically the lumen output changes at an exponential rate of -25,000/T, i.e., $L$ is proportional to $\exp (-25,000 / T)$, where $T$ is the wire temperature).

- Self-absorption, $y_{\mathrm{R}}$ : measured by operating the auxiliary lamp at a stable current and measuring the signal reported by the lumen detector. Again the value should be estimated by a minimum of 10 repeat measurements so that an uncertainty can be associated with the variable. Note: The selfabsorption coefficient, $\alpha$, is the ratio of the lumen-related signal of the test lamp, with accompanying fixturing, to that of the reference standard with its fixturing; i.e., $\alpha=y_{\mathrm{A}} / y_{\mathrm{AR}}$. Rather than using $\alpha$ in the model directly, both $y_{\mathrm{A}}$ and $y_{\mathrm{AR}}$ are used, so that the uncertainty of each is easily retained. For systems where the manufacturer provides a process for the self-absorption coefficient which is not transparent to the user, the manufacturer's software cannot be used to obtain this value and related uncertainty.

- Spectral mismatch, $F_{\mathrm{R}}$ : The ratio of the lumen signal to that from an ideal luminosity/photopic curve filter. The spectral mismatch value thus deviates from one. The detector spectral mismatch can be measured by comparing narrow band wavelength over the visible spectrum to that of a spectrally accurate detector. This is moderately difficult so the $f_{1}^{\prime}$ rating of the photometer can be used as an estimate of the mismatch and the value of the uncertainty; however, since the $f_{1}$ ' value is an average over the visible region, it applies only to white light sources and can be misleading for SSL and fluorescent lamps in particular. If the spectral mismatch is measured, it is Type A; otherwise it is a Type B value. Since most standard reference lamps have spectra that are not far from the CIE Illuminant-A spectra, the spectral mismatch function usually is small for standard lamps. For spectral detection, the spectral mismatch is assumed to have no uncertainty and is eliminated from the equation.

The following input variables are put in a separate equation model, called $C o r S_{\mathrm{R}}$, since they are described as deviations from the ideal of zero.

- Spatial mismatch, $\Delta_{s} f_{\mathrm{R}}$ : This variable expresses the deviation from a signal created by an isotropic source in an ideal sphere, i.e., one that radiates equally in every direction. It could also have been expressed as the ratio from an ideal source as for spectral mismatch, but this way of showing it is 
used in the CIE TC 2-43 work. It can be measured by measuring the signal from a collimated (small spot) source mounted at the center of a sphere and rotated over a significant number of the sphere angles. Again this is of moderate difficulty so normally it is given a Type B measurement and the value is assumed from knowledge of the sphere size and coating reflectance and the lamp angular distribution.

- Ambient Temperature Dependence, $\alpha_{\mathrm{R}} \Delta T_{\mathrm{R}}$ : Most lamps are sensitive to variations in the ambient temperature. For that reason, the integrating sphere's environmental temperature is limited to a given temperature region. The temperature dependence is shown as the product of a rate, $\alpha_{\mathrm{R}}$, and a temperature departure from nominal, $\Delta T_{\mathrm{R}}$. We assume that the temperature departure is one degree Celsius. For reference standard lamps the temperature coefficient, $\alpha_{\mathrm{R}}$, is quite small and usually can be neglected in the equation. This can be either a Type A or Type B value.

- Ageing effect, $\gamma_{\mathrm{R}} \Delta t_{\mathrm{R}}$ : Lamp lumen output is sensitive to burning time. As in the previous item, we write this as the burning time, $\Delta t_{R}$, since the lamp was obtained, times a rate of change of the lumen output with time, $\gamma_{\mathrm{R}}$. For standard reference lamps it is assumed that the lumen output will change by about $1 \%$ in 50 hours of operation. From this the rate can be established. This is then a Type B variable.

\subsubsection{Variable Estimates for the Test Lamp}

All of the input variables that apply to the reference standard lamp apply to the test lamp; however, the values obtained are obviously different. Further, the variables differ depending on the type of lamp measured and the type of equipment used. In almost all cases, the effect on lumens due to burning time is considered negligible and the operating time can be set to zero.

In the case of an incandescent lamp measured at constant current using the voltage across the resistor shunt to measure the current, the model and thus the input variables are the same as for the standard lamp. This case arises when labs make secondary standard lamps from the purchased reference standard.

For SSL or CFL replacement lamps measured in integrating spheres using spectral detection techniques, the lamp voltage is usually defined as a nominal $120 \mathrm{~V}$ so no resistor shunt is involved. The sensitivity of the lumen output to the voltage input, usually written as an exponent, $m_{\mathrm{U}}$, will also vary significantly between lamp types, even similar lamps with different ballast/driver designs. The sensitivity value can be measured by measuring the lumens at nominal voltage, a higher than nominal voltage, e.g., 125 volts, then at a lower than nominal voltage, e.g., 115 volts, and converting the differences found into an exponent by taking the log of the ratios. Further, for spectral measurements, the spectral mismatch function is assumed to be ideal, i.e., equal to one. In this case the exponent value is a Type A measurement.

Measurements of SSL fixtures or other types of reflector lamps, in integrating spheres will likely have significant error caused by the non-isotropic distribution of light. This is particularly true for integrating spheres with low $(<0.9)$ coating reflectivity. Since there may be both a non-zero average value (bias) as well as an increased uncertainty for such artifacts, one solution is to measure the lumen output on a goniometer, which has been shown by measurements at NIST and GE Lighting to be well correlated with integrating sphere measurements on other types of lamps, to obtain the value of the sphere spatial response factor, $\Delta_{s} f$. 


\subsection{Input Variables for a Mirror Goniometer}

\subsubsection{Variable Estimates for the Reference Intensity Standard}

- Luminous Intensity, $I_{\mathrm{R}}$ : given on the certificate

- Current for operation, $J_{\mathrm{R}}$ : given on certificate

- Measurement of current, $U_{\mathrm{R}}$ : same as for the integrating sphere

- Sensitivity of lumen flux to current, $m_{J_{\mathrm{R}}}$ : same as for the integrating sphere

- Self-absorption, $y_{\mathrm{R}}$ : does not apply as there is no returned radiation to the source

- Spectral Mismatch, $F_{\mathrm{R}}$ : same as for the integrating sphere

The following input variables were not included in the integrating sphere main model:

- Detector angular non-linearity, $H_{\mathrm{R}}(\theta)$ : The detector in a Type $\mathrm{C}$ goniometer (mirror or other) system receives signals from the mirror as it goes around the lamp. In the case of a rotating mirror goniometer, a cone of radiation which revolves around the axis at the mirror angle is received by the detector from the lamp through the mirror. The sensitivity of the detector to the incidence angle needs to be evaluated. For the intensity standard lamp, which is normally measured with the mirror at $90^{\circ}$ only (Illuminating Engineering Society [IES] Type C definition), the value of $H_{\mathrm{R}}$ can be viewed as that of the ratio of the signal at $90^{\circ}$ to the average signal. This factor can be measured by rotating the detector in place and recording the change of signal at various rotation angles and averaging the angular values.

- Light Distribution Sampling, $D s_{\mathrm{R}}$ : Goniometric measurements are made on the fly, i.e., the detector is gated in sync with the mirror angle so that data is collected at even intervals of the mirror angle, e.g., every $2.5^{\circ}$. Then the fixture is rotated a given amount, e.g., $22.5^{\circ}$, and the mirror angle sampling of the light intensity signal is again recorded. Since only a fraction of the space is actually sampled there is an uncertainty in the summation of lumens done from the zonal lumen data. For intensity standard lamps with a nearly isotropic distribution, this uncertainty is small: Type B variable with a nominal value of one.

The following input variables are put in a separate equation model, called $\operatorname{Cor} S_{\mathrm{R}}$, since they are described as deviations from the ideal of zero.

- Spatial mismatch, $\Delta_{\mathrm{s}} f_{\mathrm{R}}$ : does not apply as there is no reflected radiation

- Ambient Temperature Dependence, $\alpha_{\mathrm{R}} \Delta T_{\mathrm{R}}$ : same as for the integrating sphere

- Ageing effect, $\gamma_{\mathrm{R}} \Delta t_{\mathrm{R}}$ : same as for the integrating sphere

The following input variables were not included in the integrating sphere correction model equation:

- Relative distance, $\Delta d_{\mathrm{R}} / d_{\mathrm{R}}$ : positioning the reference intensity standard in the center of the mirror rotation so that the light beam to the detector distance does not change with rotation of the mirror. This input variable has some uncertainty connected with it. When using a cross-laser system to align the lamp, the uncertainty is kept to about one millimeter. Type B variable. 
- Angular tilt, $h_{\mathrm{R}}(\theta)$ : alignment of the optical axis of the lamp or fixture has some uncertainty connected with it. Normally an uncertainty of about 0.1 degree is used. Type B variable.

- Angular turn, $k_{\mathrm{R}}(\varphi)$ : alignment of the rotational plane of the lamp or fixture to the C-axis of the goniometer also has some uncertainty connected with it. Again an uncertainty of about 0.1 degree is assumed. Type B variable.

- Stray light, $S_{\mathrm{R}}(\theta)$ : The standard lamp puts light on the wall opposite to the mirror which, although painted black still reflects light back onto the mirror in such a way that it reaches the detector. The amount of stray light is difficult to measure. One way is to put a $45^{\circ}$ mirror at the wall and reflect that light into a trap which cannot reach the detector through the mirror. For most types of flat black paint, the reflected light is on the order of $0.5 \%$. Type B variable.

\subsubsection{Variable Estimates for the Test Lamp on a Goniometer}

All of the input variables that apply to the reference standard intensity lamp apply to the test lamp as discussed for the integrating sphere, but there are some important differences. Many reflector lamps or fixtures are designed to put most of the light in a down direction which is at its maximum value when the mirror angle, $\theta$ is at $0^{\circ}$ (IES Type $\mathrm{C}$ definition). Thus, an important positioning error is now with the mirror near 0 while with the standard lamp it was at $90^{\circ}$. Further, the stray light behaves very differently for the test lamp of the fixture as it is now the light bounced off the floor and received by the detector when the mirror angle is near $180^{\circ}$. For fixtures with no up-light, the stray light is easily measured, but it often can be ignored if the fixture up-light is defined as zero to begin with.

Spectral detection systems are yet not viable for the bulk of goniometer measurements so the use of a broadband detector with a photopic filter is the major focus. Thus, spectral mismatch will be just as important as it was for integrating sphere photometry, and in addition detector angular sensitivity can also be an issue if the signal is maximized for the lamp for a cone from $0^{\circ}$ while for the standard it was at $90^{\circ}$. The detector angular variability is not likely to cancel between standard and lamp or fixture measurements.

The intensity distribution from lamps or fixtures can change quite quickly with angle. Thus the error in calculating the lumen output from the sampled intensity distribution has much more uncertainty than that for sources with smooth distributions. There is not yet a robust means to evaluate the uncertainty due to a given sampling protocol, but obviously if fixtures with sharply changing light intensity are being measured, finer sampling should be done. Cameron Miller, at NIST, is studying this variable and will report on it in the future.

As discussed above, when using a reference standard calibrated in luminous intensity, the uncertainties of stray light, lamp-to-detector distance and detector angular sensitivity are likely to be important and different for the test lamp than for the intensity standard. Thus, using a calibrated incandescent/halogen reflector standard, calibrated in total lumens or face lumens (lumens in the lower hemisphere), is likely to reduce the uncertainty of measurements. No spreadsheet is developed for the case of lumen output calibration as none of the labs visited used such a procedure, so it is left as an exercise for the labs that do use this method of calibrating a goniometer. 


\subsection{Evaluate the Standard Uncertainties of Each Input Estimate}

Assume that all variables that contribute to the uncertainty of a given lamp measurement, along with their symbol and units, as well as their value, are recorded in the spreadsheet format discussed in Section 2.0. The next column shows the value of the total or standard uncertainty of each variable, followed by the type of measurement, either Type A or B.

\subsection{Type A uncertainty estimates}

Uncertainties in the photometer output signal, the auxiliary lamp photometer output signal, the voltage and current readings, and the ambient temperature difference are usually determined by experiment. When experimental methods are used, both the estimated variable value and the uncertainty are obtained from evaluation of the measured data. Note that for systems with spectral radiant measurements, the photometer reading cannot be easily separated from the calculated value of the lumen output after calibration. Thus, when referring to the photometer signal, the calculated lumen value for a given measurement normally supplied by the application software is implied. Also, note that in this case, the uncertainty of the lumen calculation is smaller than the uncertainty of the spectral radiant power given in the standard lamp uncertainty.

An example is useful in understanding how the uncertainty values are obtained in the case of selfabsorption measurements. In this case, the auxiliary lamp is turned on and allowed to stabilize, normally at a fixed current. The standard reference lamp with its associated fixturing is inserted into the sphere and a lumen/photometer reading is obtained. Then the test lamp with its associated fixturing is inserted and another lumen/photometer reading is obtained. This procedure is repeated a minimum of 10 times without turning off the auxiliary lamp. When finished, the average value for the readings with the standard reference lamp is computed as well as the standard deviation of these readings. In this case, the standard uncertainty is equal to the measured standard deviation. Similar calculations are made for the test lamp readings. Thus, from the measurements, the value of the lumen signal, the standard uncertainty, the type (A) and the degrees of freedom ( $v=N-1$, where $N$ is the number of measurements made) can be determined.

For the case of self-absorption, it is best not to turn the auxiliary lamp off during the repeat measurements, although this is allowed, as the self-absorption value is a relative value and does not depend on the stability of the auxiliary lamp output. When making repeat tests of the standard lamp and tests lamp to obtain the voltage and current readings as well as the photometer readings, it is necessary to turn off the lamp and remove it from the sphere, allowing it to cool to ambient before reinserting it for the repeat measurement.

Similarly, experimental determinations of the lumen output dependence on ambient temperature changes and voltage input changes can be measured by expanding the temperature and voltage ranges to note the effect. Spatial and spectral mismatch coefficients are more difficult but techniques for measuring both have been discussed in the literature, mostly through various NIST reports. 


\subsection{Type B Uncertainty Estimates}

The reference lamp standard comes with a calibration certificate that gives the luminous flux value or the luminous intensity value as well as the current at which it is to be operated and the combined standard uncertainty of the lumen value or the intensity value. It also gives the expanded uncertainty, usually with a coverage factor of two. The current at which the lamp is to be operated has no uncertainty connected with it. There will be an uncertainty in reading the current, but not in its nominal value. This also applies to the nominal electrical rating for the test lamp, usually the nominal lamp voltage.

Calibration certificates for the electrical instruments that measure voltage, current and power provide the center value, $c$, of the instrument for a given range. The uncertainty is often not calculated, but rather the statement is made "in tolerance." In this case, the standard uncertainty is calculated by dividing the tolerance range (often given as a percentage of range, reading and time) by the square root of 12. (See the GuUM for a discussion on how this value is obtained.) This applies to all measurements where the uncertainty is given as a range with equal probability that the reading is within that range.

The standard uncertainty of some variables that contribute to the uncertainty that are not provided by certificate values, nor easily measured, need to be obtained from experience, the literature, manufacturer's specifications, or other labs with similar systems. I will not discuss these here as each lab will have different techniques for addressing these uncertainties. In all cases, the Type B uncertainty is assumed to have infinite degrees of freedom.

\subsection{Evaluate the Covariances Associated With Input Estimates}

A correlated variable is one that changes when another related input variable is changed, i.e., it is not independent of one or more of the other inputs. In that case, the combined uncertainty (to be discussed later) needs to be calculated using special means. As far as I am aware none of the lumen measurement inputs are correlated so there is no need to go into detail on this item. If we were discussing estimating the uncertainty of color related outputs, such as the chromaticity value, then the covariance of the variables is an issue and this type of contribution needs to be investigated.

\subsection{Calculate the Result of Measurement}

On the spreadsheet, on the bottom row below the list of variables or inputs that are in the model, create a row for the output variable, i.e., for the lumen output from an integrating sphere or the luminous intensity for a goniometer. In the column for values, write the model equation by using the numbers in the cells, $x_{\mathrm{i}}$, above the cell where the output is calculated. Thus, equation 2c is solved by using the values in the cells from each row. As an example, by opening the Excel spreadsheet for "Sphere Inc-Inc" measurements, this is the equation shown in Cell E39. 


\subsection{Determine the Combined Uncertainty of the Measurement Results}

The standard uncertainty of the lumen output, $\Phi$, is obtained by appropriately combining the standard uncertainties of the input estimates as discussed above and shown in the Excel spreadsheets in column F. What is needed is to find the influence of the standard uncertainty of each variable on the lumen output. The combined standard uncertainty $u_{\mathrm{c}}(y)$ is the positive square root of the combined variance which is given by:

$$
u_{\mathrm{c}}^{2}(\Phi)=\sum_{n=1}^{n}\left(\frac{\partial f}{\partial x_{i}}\right)^{2} \cdot u^{2}\left(x_{i}\right)
$$

Here $f$ represents the lumen output value from the right-hand side of Eq. 2c. Thus, we need to take the derivative of Eq. 2c with respect to a given variable, say $y_{\mathrm{A}}$, for example, square it and multiply by the variance of that variable. Then we add together this operation for all the variables listed on the right-hand side of Eq. (2c).

The partial derivative term in Eq. (3a) is often replaced by $c_{\mathrm{i}}$, called the sensitivity coefficient, i.e.,

$$
u_{\mathrm{c}}^{2}(\Phi)=\sum_{n=1}^{n} c_{i}^{2} \cdot u^{2}\left(x_{i}\right)
$$

For spreadsheet calculations it is easier to estimate the sensitivity coefficient values by using a difference equation. Thus:

$$
c_{\mathrm{i}}=\left[f\left(x_{1}, x_{2}, \ldots x_{\mathrm{i}}+u\left(x_{\mathrm{i}}\right), \ldots x_{\mathrm{n}}\right)-f\left(x_{1}, x_{2}, \ldots x_{\mathrm{i}}-u\left(x_{\mathrm{i}}\right), \ldots x_{\mathrm{n}}\right)\right] / 2 u\left(x_{\mathrm{i}}\right)
$$

Therefore, the partial derivative (sensitivity coefficient) of the lumen output with respect to the test lamp absorption input, $y_{\mathrm{A}}$, in Eq. 2c is obtained by adding the uncertainty value, $u\left(y_{\mathrm{A}}\right)$, in column $\mathrm{F}$ for the test lamp absorption to the $y_{\mathrm{A}}$ value; then similarly subtracting the uncertainty value from the $y_{\mathrm{A}}$ term in Eq. 2c and subtracting the two modified equations, then dividing by two times the standard uncertainty of $y_{\mathrm{A}}$, i.e., $u\left(y_{\mathrm{A}}\right)$. The method given in Eq. 4 has been evaluated for all the variables and is shown in column I in all the spreadsheets. Note that the value of the sensitivity coefficient depends on the values of all the input parameters. Those that are in the denominator are negative as expected from differentiation, etc. While this method is tedious, it is very straightforward and once the equation is written in Excel, it can be copied and changed rather quickly to fill in the values for each cell. Note that if the model equation is changed, then every cell in column I must be changed to agree with the new equation.

It is now rather straightforward to calculate the combined uncertainty. In column $\mathrm{J}$, the product of the sensitivity coefficient and the standard uncertainty are calculated. These column values are called the absolute contribution of the given input to the uncertainty of the output. Thus, by calculating the sum of the squares of the values in each relevant cell in column $\mathrm{J}$ and taking the square root of the summed product, the combined uncertainty is obtained. This is shown in output variable row, i.e., the lumen or intensity outputs, in column $\mathrm{J}$.

The relative (fractional) contribution of the input variable uncertainty is shown in column K. It is simply the ratio of the absolute contribution to the estimated output. By performing the same calculation 
as in the column $\mathrm{J}$ output cell, the column $\mathrm{K}$ output cell will have the combined uncertainty as a fraction or a percentage of the output value.

\subsection{Expanded Uncertainty}

Finally, we arrive at the expanded uncertainty, $U$, which provides an interval around the output variable, lumen output, $\Phi$, in this case, which encompasses a given fraction of the distribution of values that could be expected to occur. Typically, the expanded uncertainty is obtained from the combined uncertainty by multiplying by a coverage factor, $k$. Thus:

$$
U=k \cdot u_{\mathrm{c}}(y)
$$

Normally $k$ is set equal to 2 or, if needed, 3 . For well-developed uncertainties, $k=2$, implies that about $95 \%$ confidence interval for the resulting value is obtained. For $k=3$ the confidence interval is about $99 \%$. But there is a catch.

The confidence of the values of the uncertainty depends on how many degrees of freedom are connected with the value. Hence, we need to make use of the degrees of freedom shown in column $\mathrm{H}$ to obtain an estimate of the degrees of freedom for the output variable, $\Phi$. Obviously, if the number of degrees of freedom is infinite then the uncertainty is assumed to be very well defined. On the other hand, the fewer the measurements used to establish the uncertainty of a given variable; the less likely we are to have a well-defined uncertainty of the output.

This ambiguity in the value of the standard uncertainty value influences the relationship between the coverage factor and the confidence interval. If a critical parameter uncertainty is only supported by a few measurements, then the coverage factor needed to obtain 95 percent confidence will be significantly above 2. This is taken into account with a statistical which states that the effective degrees of freedom of the lumen output are given by:

$$
v_{\mathrm{eff}}=\frac{u_{\mathrm{c}}^{4}}{\sum_{i=1}^{n} \frac{u_{i}^{4}}{v_{i}}}
$$

Here $v_{\mathrm{i}}$ are the degrees of freedom shown in column $\mathrm{H}$. What tends to occur is that the number of degrees of freedom is dominated by variables that contribute a large fraction of the absolute uncertainty. If this variable has few degrees of freedom then the effective degrees of freedom will not be much higher than that of the dominant variable. That is why it is recommended that at least ten measurements be made on each input so that the uncertainty value obtained will be somewhat well characterized.

Finally, in the column for degrees of freedom below the row for the output variable in the Excel spreadsheets is an estimate, by approximation formula from statistics, as to the coverage factor needed to obtain a coverage factor of 2, if the degrees of freedom for the output were infinite. Note that the number of degrees of freedom varies from a low of 26 for the sphere SSL setup to a high of over 31,000 for the goniometer. It is seen that a coverage factor of 2.12 is needed for the SSL case to get the same confidence level as that of 2.00 for the goniometer measurement. 


\subsection{Reporting the Results Together With Combined Uncertainty}

At this time there is no requirement from customers either in the government or industry to report the uncertainty of laboratory results on lumens or intensity. However, it has obviously been required for calibration laboratories for some time. Further, for laboratory accreditation the ISO 17025:2005 standard currently is worded such that a minimum of input factors with their uncertainty components need to be obtained and that a combined uncertainty be calculated for test laboratory accreditation. Using the spreadsheets generated through this report will allow labs to carry out these requirements in a thorough manner. 



\section{Appendix}

\section{Accompanying Excel Spreadsheet}





\section{Appendix}

\section{Accompanying Excel Spreadsheet}

The functional spreadsheet which accompanies this report is available from the DOE CALiPER program upon request. The spreadsheet follows the following principal steps, as detailed in the report:

\begin{tabular}{|c|c|}
\hline & Developing An Uncertainty Budget for Lumen Measurements Using Integrating Spheres \\
\hline Step 1 & $\begin{array}{l}\text { Write a model (mathematical equation) describing the lumen measurements of a test lamp taking into } \\
\text { account the various factors involved. } \\
\text { - use } \Phi \text { as the symbol for luminous flux of the test lamp (test lamp related quantities will not have a } \\
\text { subscript) } \\
\text { - assume measurements are taken using a broadband detector that has an output y proportional to the } \\
\text { test lamp lumen flux } \\
\text { - calibrate the system using a standard reference lamp (subscript, R) traceable to NIST } \\
\text { - from the calibration certificate for the reference standard, it is known that the reference standard } \\
\text { produces a luminous flux } \Phi_{\mathrm{R}} \text { with uncertainty, } u_{\mathrm{R}} \text {, at a fixed current } J_{\mathrm{R}} \\
\text { - thus equation from above has the form: } \Phi=\Phi_{\mathrm{R}}{ }^{*} y / y_{\mathrm{R}} \\
\text { - using an auxiliary lamp, the self-absorption of the sphere with both the test, } y_{\mathrm{A}} \text {, and reference lamp, } \\
y_{\mathrm{AR}} \text {, and their associated fixtures can be found } \\
\text { - accounting for self-absorption on the lamp lumen flux leads to the extension of the above equation } \\
\text { to the following: } \Phi=\Phi_{\mathrm{R}} *\left(y / y_{\mathrm{R}}\right)^{*}\left(y_{\mathrm{AR}} / y_{\mathrm{A}}\right) \\
\text { - determine what other factors influence the lumen output and what their contributions are }\end{array}$ \\
\hline Step 2 & $\begin{array}{l}\text { Determine all the other input variables/parameters. These are variables that will affect lumen output } \\
\text { either through its value or uncertainty } \\
\text { - uncertainty of continued operation of the standard lamp - grows with time of operation } \\
\text { - uncertainty in setting the current for the standard lamp } \\
\text { - uncertainty in measuring the current or voltage of the test lamp } \\
\text { - uncertainty in the detector response caused by differences in the angular distribution of the light } \\
\text { intensity between the test lamp and the standard lamp } \\
\text { - uncertainty in the detector response caused by differences in the spectral distribution of the light } \\
\text { intensity between the test lamp and the standard lamp } \\
\text { - uncertainty in the detector response caused by differences in the spatial distribution of the light } \\
\text { intensity between the test lamp and the standard lamp } \\
\text { - uncertainty in the actual temperature of the lamp fixture and the effect this has on lamp lumens }\end{array}$ \\
\hline $\begin{array}{l}\text { Step 3, } 4 \\
\& 6\end{array}$ & $\begin{array}{l}\text { Decide on the uncertainty values and the type of error, either Type A or Type B, represented by each } \\
\text { contribution (factor) } \\
\text { - some of the values and uncertainties can be found from calibration certificates; these are type B } \\
\text { errors with infinite degrees of freedom } \\
\text { - some values need to be measured statistically, i.e., repeat measurements, to get an average and } \\
\text { standard deviation of the measured values } \\
\text { - note that for measured values the degrees of freedom is one less than the number of measurements } \\
\text { used } \\
\text { - decide whether there is any correlated uncertainty in the measurements. If so this must be } \\
\text { accounted for although almost no measurements of lumen-related values are correlated } \\
\text { - assign a probability distribution and determine the standard uncertainty of each contribution } \\
\text { a) normal distribution: standard deviation given by } u(x) \\
\text { b) rectangular: } u\left(x_{\mathrm{i}}\right)=a_{\mathrm{i}} / \mathrm{sqrt}(3) \text { where } a_{\mathrm{i}} \text { is the half length of the interval given for the prescribed } \\
\text { limits } \\
\text { c) triangular: }\left(u\left(x_{\mathrm{i}}\right)=a_{\mathrm{i}} / \mathrm{sqrt}(6) \text { where } a_{\mathrm{i}} \text { is the half length of the interval given for the prescribed }\right. \\
\text { limits. Should be used where the majority of the values are close to the central point }\end{array}$ \\
\hline
\end{tabular}




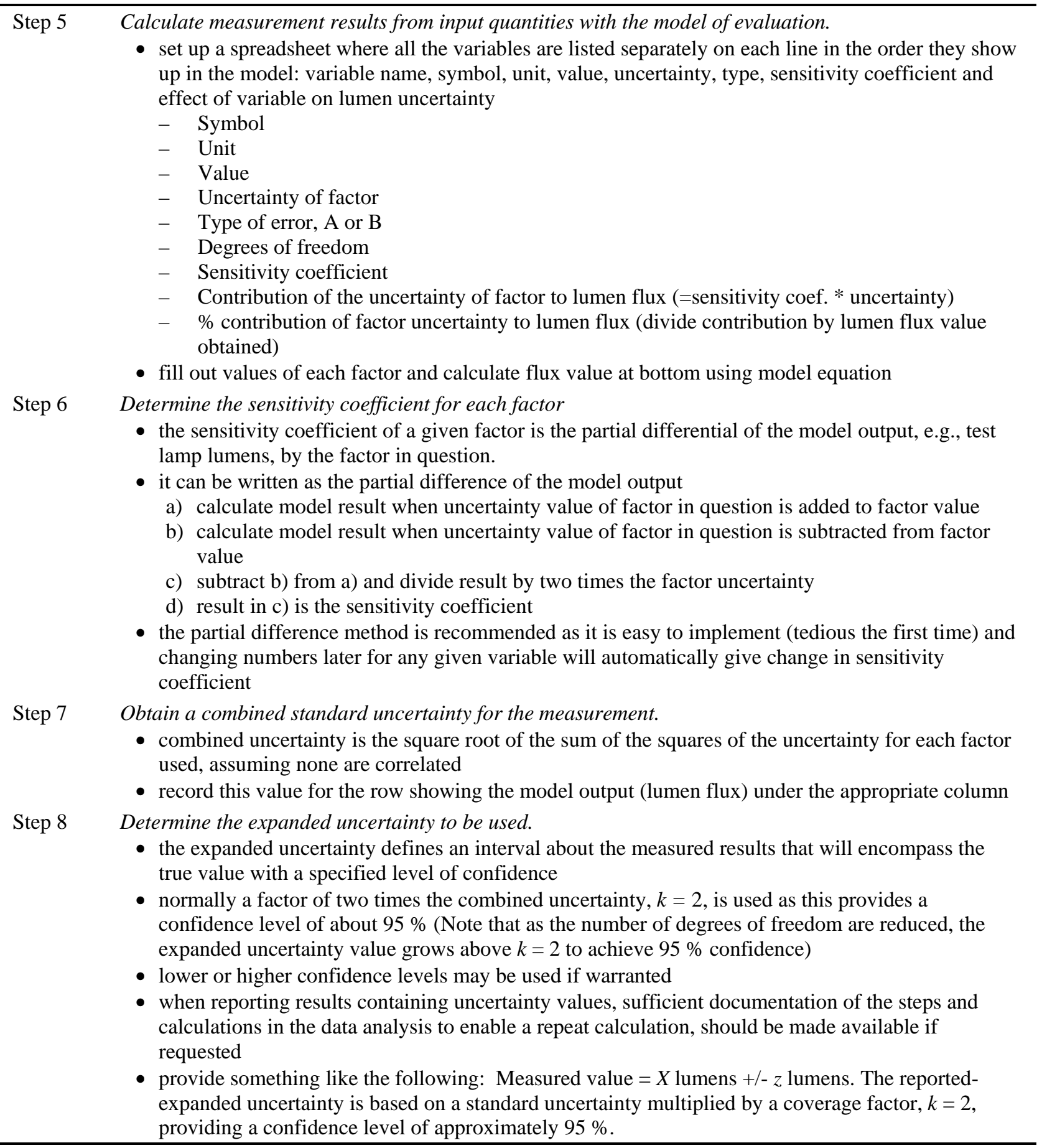

The Excel spreadsheet provides three tables enabling manual entry of values and calculation of key measurement uncertainty parameters. Examples of the spreadsheet tables are provided below in Tables A.1, A.2, and A.3. 
Table A.1. Calibration of luminous Flux by an integrating Sphere--Incandescent Reference/ Incandescent Test Lamp (secondary standard)

\begin{tabular}{|c|c|c|c|c|c|c|c|c|c|c|}
\hline No & Quantity $X_{i}$ & Symbol & Unit & $\begin{array}{c}\text { Value } \\
x_{i}\end{array}$ & $\begin{array}{c}\text { Abs. std. } \\
\text { uncertainty } \\
u\left(x_{i}\right)\end{array}$ & Type & $\begin{array}{c}\text { DOF } \\
v\end{array}$ & $\begin{array}{c}\text { Abs. } \\
\text { sensitivity } \\
c_{i}\end{array}$ & $\begin{array}{c}\text { Abs. } \\
\text { contribution } \\
u_{i}(y)\end{array}$ & $\begin{array}{c}\text { Relative } \\
\text { contribution } \\
\mathrm{u}_{\mathrm{rel}}(\mathrm{y})\end{array}$ \\
\hline 3 & sphere spatial response factor -test la & $\Delta_{S} f$ & & 0.001 & 0.0012 & B & $\infty$ & -1.0000 & -0.00120 & -0.00120 \\
\hline 4 & relative aging coeff.-test lamp & $\gamma$ & $1 / \mathrm{h}$ & 0.0003 & 0.0003 & B & $\infty$ & -0.1000 & -0.00003 & -0.00003 \\
\hline 5 & burning time-test lamp & $\Delta t$ & $\mathrm{~h}$ & 0.10 & 0 & B & $\infty$ & & & \\
\hline 6 & correction factor -test lamp & $\operatorname{corS}$ & & 0.9990 & & & $\infty$ & & 0.00120 & 0.0012 \\
\hline 9 & sphere spatial response factor-REF & $\Delta_{S} f_{R}$ & & 0.000 & 0.00050 & B & $\infty$ & -1.000 & -0.00050 & -0.00050 \\
\hline 14 & relative aging coeff.-REF & $\gamma_{R}$ & $1 / \mathrm{h}$ & 0.0002 & 0.0001 & $\mathrm{~B}$ & $\infty$ & -10.000 & -0.00100 & -0.00100 \\
\hline 15 & burning time-REF & $\Delta t_{R}$ & $\mathrm{~h}$ & 10.00 & 0 & $\mathrm{~B}$ & $\infty$ & & & \\
\hline 12 & correction factor-REF & $\operatorname{corS}_{R}$ & & 0.9980 & & & $\infty$ & & 0.00112 & 0.00112 \\
\hline 13 & correction factor-REF & $\operatorname{corS}_{R}$ & & 0.9980 & 0.00112 & A & $\infty$ & 7026.4 & 7.855739 & 0.001120 \\
\hline 14 & correction factor-test lamp & $\operatorname{corS}$ & & 0.9990 & 0.00120 & $\mathrm{~A}$ & $\infty$ & -7019.6 & -8.426121 & -0.001202 \\
\hline 15 & luminous flux-REF & $\Phi_{R}$ & $\operatorname{Im}$ & 2800.0 & 4.800 & $\mathrm{~B}$ & $\infty$ & 2.504 & 12.021144 & 0.001714 \\
\hline 16 & photometer-source & $y$ & $\mathrm{~V}$ & 4.579 & 0.0085 & A & 9 & 1531.4 & 13.017000 & 0.001856 \\
\hline 17 & photometer-REF & $y_{R}$ & $\mathrm{~V}$ & 1.765 & 0.0007 & A & 9 & -3973.0 & -2.781096 & -0.000397 \\
\hline 18 & photometer AUX-test lamp & $y_{A}$ & $\mathrm{~V}$ & 3.1234 & 0.001 & A & 9 & -2245.1 & -2.245097 & -0.000320 \\
\hline 19 & photometer AUX-REF & $y_{A R}$ & $\mathrm{~V}$ & 3.1243 & 0.001 & $\mathrm{~A}$ & 9 & 2244.4 & 2.244450 & 0.000320 \\
\hline 20 & shunt voltage-test lamp & $U$ & $\mathrm{~V}$ & 0.88000 & 0.00012 & $\mathrm{~A}$ & 9 & -55461.2 & -6.655344 & -0.000949 \\
\hline 21 & shunt voltage-REF & $U_{R}$ & $\mathrm{~V}$ & 0.58000 & 0.000075 & $\mathrm{~A}$ & 9 & 86082.5 & 6.456184 & 0.000921 \\
\hline 22 & current-lumen exponent-test lamp & $m_{J}$ & & 6.96 & 0.38 & $\mathrm{~B}$ & $\infty$ & -3.284 & -1.248087 & -0.000178 \\
\hline 23 & current-lumen exponent-REF & $m_{J R}$ & & 7.12 & 0.22 & $\mathrm{~B}$ & $\infty$ & -30.862 & -6.789553 & -0.000968 \\
\hline 26 & spetral mismatch-test lamp & $F$ & & 1 & 0.001 & $\mathrm{~B}$ & $\infty$ & -7012.3 & -7.012341 & -0.001000 \\
\hline 27 & spectral mismatch-REF & $F_{R}$ & & 1 & 0.0005 & $\mathrm{~B}$ & $\infty$ & 7012.3 & 3.506167 & 0.000500 \\
\hline 28 & shunt resistor & $R$ & Ohm & 0.100042 & 0.000006 & $\mathrm{~B}$ & $\infty$ & -499068.6 & -2.994412 & -0.000427 \\
\hline 29 & cal. factor current DVM & $c$ & & 1.00032 & 0.000041 & $\mathrm{~B}$ & $\infty$ & 559.96 & 0.022958 & 0.000003 \\
\hline 32 & nominal current-source & $J$ & A & 8.7950 & 0 & & & & & \\
\hline 33 & nominal current-REF & $J_{R}$ & $\mathrm{~A}$ & 5.8250 & 0 & & & & & 0.002631 \\
\hline 34 & luminous flux - test lamp & $\Phi_{1}$ & $\mathrm{Im}$ & 7012.3 & & & 124 & & 25.86 & 0.0037 \\
\hline & & & & & & & & & & \\
\hline & & & \multicolumn{4}{|c|}{ found from approximation formula: $k=$} & 2.03 & & 52.5 & 0.0075 \\
\hline & & & & & & & & & & \\
\hline & & & & & & & \multicolumn{3}{|c|}{ variance - Test Lamp $u_{\text {dev }}(y)=$} & $0.26 \%$ \\
\hline & & & & & & & \multicolumn{2}{|c|}{ variance - REF } & $u_{\text {inst }}(y)=$ & $0.26 \%$ \\
\hline
\end{tabular}


Table A.2. Measurement of Luminous Flux by an Integrating sphere-Incandescent Reference / SSL or CFL Test Lamp

\begin{tabular}{|c|c|c|c|c|c|c|c|c|c|c|}
\hline No & Quantity $X_{i}$ & Symbol & Unit & $\begin{array}{c}\text { Value } \\
x_{i}\end{array}$ & $\begin{array}{c}\text { Abs. std. } \\
\text { uncertainty } \\
u\left(x_{i}\right)\end{array}$ & Type & $\begin{array}{c}\text { DOF } \\
v\end{array}$ & $\begin{array}{l}\text { Abs. sensitivity } \\
\qquad \mathrm{c}_{\mathrm{i}}\end{array}$ & $\begin{array}{c}\text { Abs. } \\
\text { contribution } \\
u_{i}(y)\end{array}$ & $\begin{array}{c}\text { Relative } \\
\text { contribution } \\
\mathrm{u}_{\mathrm{rel}}(\mathrm{y})\end{array}$ \\
\hline 1 & ambient temp. difference & $\Delta T a$ & ${ }^{\circ} \mathrm{C}$ & 2.0 & 1.0 & A & 29 & 0.0020 & 0.00200 & 0.00199 \\
\hline 2 & ambient temp. coeff. & $\alpha$ & $1 / \mathrm{K}$ & 0.002 & 0.001 & A & 29 & 2.0000 & 0.00200 & 0.00199 \\
\hline 3 & sphere spatial response factor & $\Delta_{s} f$ & & 0.0 & 0.0012 & B & $\infty$ & -1.0000 & -0.00120 & -0.00120 \\
\hline 4 & stray light & $S(\theta)$ & & 0.0 & 0.0050 & B & $\infty$ & -1.0000 & -0.00500 & -0.00498 \\
\hline 5 & relative aging coeff. & $\gamma$ & $1 / \mathrm{h}$ & 0.0003 & 0.0003 & $\mathrm{~B}$ & $\infty$ & -0.1000 & -0.00003 & -0.00003 \\
\hline 6 & burning time & $\Delta t$ & $\mathrm{~h}$ & 0.10 & 0 & $\mathrm{~B}$ & $\infty$ & & & \\
\hline 7 & correction factor -test lamp & $\operatorname{corS}$ & & 1.0040 & & & $2.15 \mathrm{E}+03$ & & 0.00587 & 0.0058 \\
\hline 8 & ambient temp. difference-REF & $\Delta T_{\alpha R}$ & ${ }^{\circ} \mathrm{C}$ & 2.0 & 1.0 & $\mathrm{~A}$ & \begin{tabular}{|l|}
29 \\
\end{tabular} & 0.00015 & 0.00015 & 0.00015 \\
\hline 9 & ambient temp. coeff. -REF & $\alpha_{R}$ & $1 / K$ & 0.00015 & 0.00002 & $\mathrm{~B}$ & $\infty$ & 2.000 & 0.00004 & 0.00004 \\
\hline 10 & sphere spatial response factor-REF & $\Delta_{S} f_{R}$ & & 0.0 & 0.00050 & $\mathrm{~B}$ & $\infty$ & -1.000 & -0.00050 & -0.00050 \\
\hline 11 & stray light-REF & $S_{R}(\theta)$ & & 0.0 & 0.00200 & $\mathrm{~B}$ & $\infty$ & -1.000 & -0.00200 & -0.00200 \\
\hline 12 & relative aging coeff.-REF & $\gamma_{R}$ & $1 / \mathrm{h}$ & 0.0002 & 0.0001 & $\mathrm{~B}$ & $\infty$ & -2.000 & -0.00020 & -0.00020 \\
\hline 13 & burning time-REF & $\Delta t_{R}$ & $\mathrm{~h}$ & 2.00 & 0 & B & $\infty$ & & & \\
\hline 14 & correction factor-REF & $\operatorname{corS}_{R}$ & & 0.9999 & & & 1066138 & & 0.00208 & 0.00208 \\
\hline 15 & correction factor-REF & $\operatorname{corS}_{R}$ & & 0.9999 & 0.00208 & A & 1066138 & 909.44 & 1.888946 & 0.002077 \\
\hline 16 & correction factor-test lamp & $\operatorname{corS}$ & & 1.0040 & 0.00587 & $\mathrm{~A}$ & $2.15 \mathrm{E}+03$ & -905.78 & -5.315723 & -0.005846 \\
\hline 17 & luminous flux-REF & $\Phi_{R}$ & $\mathrm{Im}$ & 1715.0 & 8.600 & $\mathrm{~B}$ & $\infty$ & 0.53023 & 4.560005 & 0.005015 \\
\hline 18 & photometer-test lamp & $y$ & Im & 920 & 9.6 & A & 9 & 0.988 & 9.488867 & 0.010435 \\
\hline 19 & photometer-REF & $y_{R}$ & $\mathrm{Im}$ & 1715.0 & 5.0 & $\mathrm{~A}$ & 9 & -0.530 & -2.651188 & -0.002915 \\
\hline 20 & photometer - AUX test lamp & $y_{A}$ & $\operatorname{Im}$ & 790.0 & 2.0 & A & 9 & -1.151 & -2.302166 & -0.002532 \\
\hline 21 & photometer-AUX REF & $y_{A R}$ & Im & 805.0 & 1.0 & A & 9 & 1.130 & 1.129627 & 0.001242 \\
\hline 22 & measured voltage-test lamp & $U$ & $\mathrm{~V}$ & 119.99 & 0.012 & A & 29 & 0.0 & 0.000095 & 0.000000 \\
\hline 23 & cal. factor AC voltage & $c_{U}$ & & 0.9986 & 0.00104 & $\mathrm{~B}$ & $\infty$ & 910.62 & 0.947050 & 0.001041 \\
\hline 24 & voltage-lumen expoent - test lamp & $m_{U}$ & & 0.5 & 0.1 & A & 9 & & & \\
\hline 25 & shunt voltage-REF & $U_{R}$ & $\mathrm{~V}$ & 0.08490 & 0.000006 & A & 29 & 76261.14 & 0.457567 & 0.000503 \\
\hline 26 & cal. factor DC voltage & $c_{R}$ & & 0.9965 & 0.000043 & $\mathrm{~B}$ & $\infty$ & 6497.31 & 0.279384 & 0.000307 \\
\hline 27 & shunt resistor & $R$ & Ohm & 0.099999 & 0.000071 & $\mathrm{~B}$ & $\infty$ & -64746.62 & -4.597010 & -0.005055 \\
\hline 28 & current-lumen exponent-REF & $m_{J R}$ & & 7.12 & 0.22 & $\mathrm{~B}$ & $\infty$ & -3.18103 & -0.699827 & -0.000770 \\
\hline 29 & nominal voltage - source & $U_{0}$ & V & 120.00 & 0 & & & & & \\
\hline 30 & nominal current-REF & $J_{R}$ & A & 0.8490 & 0 & & & & & 0.012226 \\
\hline 29 & luminous flux- test lamp & $\Phi$ & $\operatorname{Im}$ & 909.3 & & & 35 & & 13.38 & 0.0147 \\
\hline & & & \multicolumn{4}{|c|}{ found from approximation formula: $k=$} & 2.09 & & 28.0 & 0.0308 \\
\hline & & & & & & & & & & \\
\hline & & & & & & & & \multicolumn{2}{|l|}{ variance - Test Lamp } & $1.22 \%$ \\
\hline & & & & & & & & \begin{tabular}{|l|l} 
variance - REF \\
\end{tabular} & $u_{\text {inst }}(y)=$ & $0.82 \%$ \\
\hline
\end{tabular}


Table A.3. Measurement of Luminous Intensity by a Goniometer-Incandescent Reference / Any Type of Test Lamp

\begin{tabular}{|c|c|c|c|c|c|c|c|c|c|c|}
\hline No & Quantity $X_{i}$ & Symbol & Unit & $\begin{array}{c}\text { Value } \\
x_{i}\end{array}$ & $\begin{array}{l}\text { Abs. std. } \\
\text { uncertainty } \\
u\left(x_{i}\right)\end{array}$ & Type & $\begin{array}{c}\text { DOF } \\
v\end{array}$ & $\begin{array}{c}\text { Abs. } \\
\text { sensitivity } \\
\mathrm{c}_{\mathrm{i}}\end{array}$ & $\begin{array}{c}\text { Abs. } \\
\text { contribution } \\
u_{i}(y)\end{array}$ & $\begin{array}{c}\text { Relative } \\
\text { contribution } \\
\mathrm{u}_{\mathrm{rel}}(\mathrm{y})\end{array}$ \\
\hline 1 & amb.temp. difference - test lamp & $\Delta T a$ & ${ }^{\circ} \mathrm{C}$ & 2.0 & 1.0 & $\mathrm{~A}$ & 29 & 0.0007 & 0.00070 & 0.00070 \\
\hline 2 & rel.temp.coeff. - test lamp & $\alpha$ & $1 / K$ & 0.0007 & 0.0003 & B & $\infty$ & 2.000 & 0.00060 & 0.00060 \\
\hline 3 & relative dist. variation - test lamp & $\Delta d / d$ & & 0.0 & 0.000300 & B & $\infty$ & 2.000 & 0.00060 & 0.00060 \\
\hline 4 & angular tilt - test lamp & $h(\theta)$ & & 0.0 & 0.001500 & $\mathrm{~B}$ & $\infty$ & 1.000 & 0.00150 & 0.00150 \\
\hline 5 & angular turn - test lamp & $k(\varphi)$ & & 0.0 & 0.000650 & $\mathrm{~B}$ & $\infty$ & 1.000 & 0.00065 & 0.00065 \\
\hline 7 & stray light - test lamp & $S(\theta)$ & & 0.001 & 0.001 & $\mathrm{~B}$ & $\infty$ & -1.000 & -0.00100 & -0.00100 \\
\hline 6 & rel.aging coeff. - test lamp & $\gamma$ & $1 / \mathrm{h}$ & 0.0003 & 0.0003 & $\mathrm{~B}$ & $\infty$ & -0.100 & -0.00003 & -0.00003 \\
\hline 8 & burning time - test lamp & $\Delta t$ & $\mathrm{~h}$ & 0.10 & 0 & $B$ & $\infty$ & & & \\
\hline 9 & correction factor - test lamp & $\operatorname{corS}$ & & 1.0004 & & & 2,880 & & 0.00221 & 0.00221 \\
\hline 10 & amb.temp. difference - REF & $\Delta T_{\alpha R}$ & ${ }^{\circ} \mathrm{C}$ & 2.0 & 1.0 & $\mathrm{~A}$ & 29 & 0.00015 & 0.00015 & 0.00003 \\
\hline 11 & rel.temp.coeff. - REF & $\alpha_{R}$ & $1 / K$ & 0.00015 & 0.00002 & $\mathrm{~B}$ & $\infty$ & 2.0000 & 0.00004 & 0.00000 \\
\hline 12 & relative distance variation - REF & $\Delta d_{R} / d_{R}$ & & 0.0 & 0.00050 & $\mathrm{~B}$ & $\infty$ & 2.0000 & 0.00100 & 0.00101 \\
\hline 13 & angular tilt - REF & $h_{R}(\theta)$ & & 0.0 & 0.00020 & $B$ & $\infty$ & 1.0000 & 0.00020 & 0.00023 \\
\hline 14 & angular turn - REF & $k_{R}(\varphi)$ & & 0.0 & 0.00040 & $\mathrm{~B}$ & $\infty$ & 1.0000 & 0.00040 & 0.00040 \\
\hline 16 & stray light - REF & $S_{R}(\theta)$ & & 0.005 & 0.001 & $B$ & $\infty$ & -1.0000 & -0.00100 & 0.00000 \\
\hline 15 & rel.aging coeff. - REF & $\gamma_{R}$ & $1 / \mathrm{h}$ & 0.0002 & 0.0001 & $B$ & $\infty$ & -5.0000 & -0.00050 & -0.00050 \\
\hline 17 & burning time - REF & $\Delta t_{R}$ & $\mathrm{~h}$ & 5.00 & 0 & $\mathrm{~B}$ & $\infty$ & & & \\
\hline 18 & correction factor source - REF & $\operatorname{corS}_{R}$ & & 0.9943 & & & 350,645 & & 0.00157 & 0.00023 \\
\hline 19 & correction factor - REF & $\operatorname{corS} S_{R}$ & & 0.9943 & 0.001573 & A & 350,645 & 672.5 & 1.05785 & 0.00158 \\
\hline 20 & correction factor -test lamp & $\operatorname{corS}$ & & 1.0004 & 0.002210 & $A$ & 2,880 & -668.5 & -1.47718 & -0.00221 \\
\hline 21 & luminous intensity - REF & $I_{R}$ & $\mathrm{~cd}$ & 280.00 & 0.680 & B & $\infty$ & 2.388 & 1.62399 & 0.00243 \\
\hline 22 & photometer signal - test lamp & $y$ & V & 4.579 & 0.0085 & $A$ & 9 & 146.0 & 1.24131 & 0.00186 \\
\hline 23 & photometer signal - REF & $y_{R}$ & $\mathrm{~V}$ & 1.765 & 0.0007 & A & 9 & -378.9 & -0.26521 & -0.00040 \\
\hline 24 & shunt voltage & $U$ & $\mathrm{~V}$ & 0.88000 & 0.00012 & A & 9 & -5289 & -0.63466 & -0.00095 \\
\hline 25 & shunt voltage - REF & $U_{R}$ & $\mathrm{~V}$ & 0.58000 & 0.000075 & A & 9 & 8209 & 0.61567 & 0.00092 \\
\hline 26 & current-intensity exponent & $m_{J}$ & & 6.96 & 0.38 & $\mathrm{~B}$ & $\infty$ & -0.3132 & -0.11902 & -0.00018 \\
\hline 27 & current-intensity exponent - REF & $m_{J R}$ & & 7.12 & 0.22 & B & $\infty$ & -2.9430 & -0.64746 & -0.00097 \\
\hline 28 & Spectral mismatch - test lamp & $F$ & $\mathrm{~K}$ & 2900 & 25 & $B$ & $\infty$ & -0.2306 & -5.76509 & -0.00862 \\
\hline 29 & Spectral mismatch - REF & $F_{R}$ & $\mathrm{~K}$ & 2800 & 10 & $\mathrm{~B}$ & $\infty$ & 0.2388 & 2.38822 & 0.00357 \\
\hline 30 & detector non-linearity-test lamp & $H(\theta)$ & & 1.002 & 0.002 & A & 9 & -667.4 & -1.33474 & -0.00200 \\
\hline 31 & detector non-linearity-REF & $H_{R}(\theta)$ & & 0.995 & 0.002 & A & 9 & 672.1 & 1.34412 & 0.00201 \\
\hline 32 & Distribution sample - test lamp & Ds & & 1.000 & 0.01 & $\mathrm{~B}$ & $\infty$ & -668.8 & -6.68767 & -0.01000 \\
\hline 33 & Distribution sample - REF & $D s_{R}$ & & 1.000 & 0.001 & B & $\infty$ & 668.7 & 0.66870 & 0.00100 \\
\hline 34 & shunt resistor & $R$ & Ohm & 0.100042 & 0.000006 & $B$ & $\infty$ & -1069 & -0.00642 & -0.00001 \\
\hline 35 & cal. factor - DVM & $c$ & & 1.00032 & 0.000041 & $\mathrm{~B}$ & $\infty$ & 107.0 & 0.00439 & 0.00001 \\
\hline 36 & nominal current of test lamp & $J$ & A & 8.7950 & 0 & & & & & \\
\hline 37 & nominal current - REF & $J_{R}$ & A & 5.8250 & 0 & & & & & 0.01355 \\
\hline 38 & intensity at nominal current & $I$ & $\mathrm{~cd}$ & 668.7 & & & 31,018 & & 9.8217 & 0.0147 \\
\hline & & & \multicolumn{4}{|c|}{ found from approximation formula: $k=$} & 2.00 & & 19.6 & 0.0294 \\
\hline & & & & & & & & & & \\
\hline & & & & & & & & variance & test lamp & $1.36 \%$ \\
\hline & & & & & & & & variance & REF & $0.57 \%$ \\
\hline
\end{tabular}




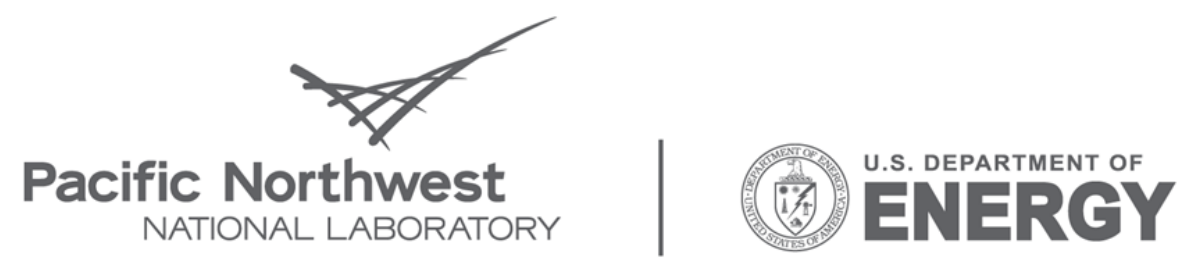

Proudly Operated by Battelle Since 1965

902 Battelle Boulevard

P.O. Box 999

Richland, WA 99352

1-888-375-PNNL (7665)

www.pnl.gov 